\title{
On minimal periods of functional-differential equations and difference inclusions
}

\author{
by M. MEDVEĎ (Bratislava)
}

\begin{abstract}
We prove several results on lower bounds for the periods of periodic solutions of some classes of functional-differential equations in Hilbert and Banach spaces and difference inclusions in Hilbert spaces.

Introduction. In this paper we give a simple method for finding lower bounds for the periods of periodic solutions of some classes of functionaldifferential equations and difference inclusions in Hilbert spaces. First results on lower bounds of differential and functional-differential equations were proved by J. A. Yorke [8], A. Lasota and J. A. Yorke [4] and T. Y. Li [5]. Recently W. Słomczyński [7] gave a generalization of Theorem 4 of Lasota and Yorke [4] to delay differential equations of the form
\end{abstract}

$$
\dot{x}(t)=F(x(t), x(\tau(t)), t) .
$$

He also studied this problem for difference equations of the form

$$
x_{n+1}-x_{n}=f\left(x_{n}, x_{n-1}, n\right) .
$$

His method differs from that used in the above mentioned papers. The problem of the existence of a lower bound for the periods of periodic solutions of difference equations has also been studied by S. Busenberg, M. Martelli and D. Fisher (see [1]-[3]).

We give a new approach to the problem of finding a lower bound for the periods of periodic solutions of functional-differential equations which we apply to equations of the form

$$
\dot{x}(t)=f_{1}\left(x\left(\tau_{1}(t)\right)\right)+\ldots+f_{m}\left(x\left(\tau_{m}(t)\right)\right) .
$$

If we take the equation (3) with $\tau_{1}(t) \equiv t, f_{3}, \ldots, f_{m} \equiv 0$ we obtain an equation of the form (1). Our result concerning the latter equation is weaker than that proved in [7]. Probably it is possible to prove a stronger result concerning (3) with several delays by another method; however, our method is simple and we shall show that it is also suitable for a class of difference inclusions. 
We also prove some results on functional-differential equations by the method developed in [1]-[3].

\section{Bounds for periods of functional-differential equations}

TheOREM 1. Let $H$ be a Hilbert space, let $f_{i}: H \rightarrow H, i=1, \ldots, m$, be Lipschitz mappings with Lipschitz constant $L>0$ and let $\tau_{i}: \mathbb{R} \rightarrow \mathbb{R}$, $i=1, \ldots, m$, be continuously differentiable, strictly monotone functions. If the equation (3) has a nonconstant, T-periodic solution then

$$
T \geq 2 / K L m \text {, }
$$

where $K=K_{1} K_{2}, K_{1}=\max \left\{\left|\dot{\tau}_{i}(t)\right|^{-1}: 0 \leq t \leq T, 1 \leq i \leq m\right\}, K_{2}=$ $\max \left\{\left|\dot{\tau}_{i}(s)\right|: 0 \leq s \leq T, 1 \leq i \leq m\right\}$.

Proof. Let $\varphi(t)$ be a $T$-periodic, nonconstant solution of $(3)$ and $d=$ $\max \{\|\varphi(t)-\varphi(s)\|: 0 \leq t, s \leq T\}$, where $\|u\|=(u, u)^{1 / 2},(\cdot, \cdot)$ is the scalar product in $H$. Then there exist $x_{0}, y_{0} \in \gamma:=\{\varphi(t): 0 \leq t \leq T\}$ such that $d=\left\|x_{0}-y_{0}\right\|$. Obviously, there are $t_{1}, t_{2} \in[0, T]$ such that $\varphi\left(t_{1}\right)=x_{0}$, $\varphi\left(t_{2}\right)=y_{0}$ (we assume $\left.t_{1}<t_{2}\right)$, and so

$$
\begin{aligned}
d^{2} & =\left(y_{0}-x_{0}, y_{0}-x_{0}\right)=\left(y_{0}-x_{0}, \varphi\left(t_{2}\right)-\varphi\left(t_{1}\right)\right) \\
& =\left(y_{0}-x_{0}, \int_{t_{1}}^{t_{2}}\left(f_{1}\left(\varphi\left(\tau_{1}(s)\right)\right)+\ldots+f_{m}\left(\varphi\left(\tau_{m}(s)\right)\right)\right) d s\right) .
\end{aligned}
$$

Thus

$$
d^{2}=\sum_{i=1}^{m} \int_{t_{1}}^{t_{2}}\left(y-x_{0}, f_{i}\left(\varphi\left(\tau_{i}(s)\right)\right)\right) d s
$$

Since $\tau_{i} \in C^{1}$ and it is strictly monotone, we have

$$
\begin{aligned}
\int_{t_{1}}^{t_{2}}\left(y_{0}-\right. & \left.x_{0}, f_{i}\left(\varphi\left(\tau_{i}(s)\right)\right)\right) d s=\int_{\tau_{i}\left(t_{1}\right)}^{\tau_{i}\left(t_{2}\right)}\left(y_{0}-x_{0}, f_{i}(\varphi(r))\left(\dot{\tau}_{i}\left(\tau_{i}^{-1}(r)\right)\right)^{-1} d r\right. \\
& \leq \max _{x \in \gamma}\left(y_{0}-x_{0}, f_{i}(x)\right)\left|\int_{\tau_{i}\left(t_{1}\right)}^{\tau_{i}\left(t_{2}\right)}\left(\dot{\tau}_{i}\left(\tau_{i}^{-1}(r)\right)\right)^{-1} d r\right| \\
& \leq \max _{x \in \gamma}\left(y_{0}-x_{0}, f_{i}(x)\right) \cdot \max _{0 \leq t \leq T}\left(\left|\dot{\tau}_{i}(t)\right|^{-1}\right)\left|\tau_{i}\left(t_{2}\right)-\tau_{i}\left(t_{1}\right)\right| \\
& \leq \max _{x \in \gamma}\left(y_{0}-x_{0}, f_{i}(x)\right) \cdot \max _{0 \leq t \leq T}\left(\left|\dot{\tau}_{i}(t)\right|^{-1}\right) \cdot \max _{0 \leq s \leq T}\left|\dot{\tau}_{i}(s)\right|\left|t_{2}-t_{1}\right| .
\end{aligned}
$$

Thus we have proved that

$$
d^{2} \leq K T \sum_{i=1}^{m} \max _{x \in \gamma}\left(y_{0}-x_{0}, f_{i}(x)\right),
$$


where $K$ is defined as in the theorem.

Let $\max _{x \in \gamma}\left(y_{0}-x_{0}, f_{i}(x)\right)=\left(y_{0}-x_{0}, f_{i}\left(x_{i}\right)\right)$ for some $x_{i} \in \gamma$. Then we can write (6) in the form

$$
d^{2} \leq K T \sum_{i=1}^{m}\left(y_{0}-x_{0}, f_{i}\left(x_{i}\right)\right) .
$$

If we change the roles of $x_{0}$ and $y_{0}$ we obtain

$$
d^{2} \leq K T \sum_{i=1}^{m}\left(x_{0}-y_{0}, f_{i}\left(y_{i}\right)\right)
$$

for some $y_{i} \in \gamma$. From (7), (8) we get

$$
\begin{aligned}
2 d^{2} & \leq K T\left(y_{0}-x_{0}, \sum_{i=1}^{m}\left(f_{i}\left(x_{i}\right)-f_{i}\left(y_{i}\right)\right)\right) \leq K T\left\|y_{0}-x_{0}\right\| \sum_{i=1}^{m} L\left\|x_{i}-y_{i}\right\| \\
& \leq K L T m\left\|y_{0}-x_{0}\right\|^{2}=K L T m d^{2} .
\end{aligned}
$$

This inequality immediately yields (4).

Now consider the equation

$$
\frac{d^{n} x(t)}{d t^{n}}=F\left(x\left(\tau_{1}(t)\right), \ldots, x\left(\tau_{m}(t)\right)\right), \quad x \in B,
$$

where $B$ is a Banach space. As a direct consequence of [3, Lemma 3.1] we obtain

Lemma. Let $B$ be a Banach space and let $y: \mathbb{R} \rightarrow B$ be a T-periodic mapping of class $C^{n-1}$ with $\left\|y^{(n)}(t)\right\|$ integrable. Then

$$
\int_{0}^{T} \int_{0}^{T}\|y(t)-y(s)\| d s d t \leq(T / 6)^{n} \int_{0}^{T} \int_{0}^{T}\left\|y^{(n)}(t)-y^{(n)}(s)\right\| d s d t
$$

$\left(y^{(n)}:=d^{n} y / d t^{n}\right)$.

Theorem 2. Let $B$ be a Banach space, let $F: B \times \ldots \times B \rightarrow B$ satisfy the Lipschitz condition

$$
\left\|F\left(x_{1}, \ldots, x_{m}\right)-F\left(y_{1}, \ldots, y_{m}\right)\right\| \leq L \sum_{i=1}^{m}\left\|x_{i}-y_{i}\right\|
$$

for all $x_{i}, y_{i} \in B$, and let $\tau_{i}: \mathbb{R} \rightarrow \mathbb{R}, i=1, \ldots, m$, be continuously differentiable, strictly monotone functions with $\left|\dot{\tau}_{i}(t)\right| \leq 1$ for all $t \in \mathbb{R}$. If the equation (9) has a nonconstant, T-periodic solution $x(t)$ then

$$
T \geq 6(L m)^{-1 / n} .
$$


Proof. The Lemma and (10) yield

$$
\begin{aligned}
\int_{0}^{T} \int_{0}^{T}\|x(t)-x(s)\| d s d t & \leq L(T / 6)^{n} \sum_{i=1}^{m} \int_{0}^{T} \int_{0}^{T}\left\|x\left(\tau_{i}(t)\right)-x\left(\tau_{i}(s)\right)\right\| d s d t \\
& \leq L(T / 6)^{n} \sum_{i=1}^{m} \int_{\tau_{i}(0)}^{\tau_{i}(T)} \int_{\tau_{i}(0)}^{\tau_{i}(T)} \| x(p)-x(q) \mid d p d q \\
& \leq m L(T / 6)^{n} \int_{0}^{T} \int_{0}^{T}\|x(p)-x(q)\| d p d q .
\end{aligned}
$$

This implies that $m L(T / 6)^{n} \geq 1$ and (11) follows.

Now we use the above lemma to solve the problem of finding a lower estimate for the periods of periodic solutions of an equation of the form

$$
\dot{x}(t)=G\left(x(t), x^{2}(t), \ldots, x^{m}(t)\right),
$$

where $G: \mathbb{R}^{m} \rightarrow \mathbb{R}, x: \mathbb{R} \rightarrow \mathbb{R}$, and $x^{i}(t)=(x \circ \ldots \circ x)(t)$ is the $i$ th iteration of $x$.

Theorem 3. Let $G: \mathbb{R}^{m} \rightarrow \mathbb{R}$ satisfy the Lipschitz condition

$$
\left|G\left(x_{1}, \ldots, x_{m}\right)-G\left(y_{1}, \ldots, y_{m}\right)\right| \leq L \sum_{i=1}^{m}\left|x_{i}-y_{i}\right|
$$

for all $x_{i}, y_{i} \in \mathbb{R}$ and suppose there is a constant $M>0$ such that $|G(u)| \leq$ $M$ for all $u \in \mathbb{R}^{m}$. If the equation (12) has a nonconstant, T-periodic solution $x(t)$ then

$$
\begin{array}{ll}
T \geq 6\left(M^{m-1}-1\right)((M-1) L)^{-1} & \text { if } M \neq 1, \\
T \geq 6(L m)^{-1} & \text { if } M=1 .
\end{array}
$$

We shall formulate and prove a more general theorem concerning functional-differential equations of the form appearing in ecological models (see e.g. [6]).

Consider the functional-differential equation

$$
\dot{x}(t)=g\left(J^{k_{1}}\left(G_{1} \circ x\right)(t), \ldots, J^{k_{m}}\left(G_{m} \circ x\right)(t)\right),
$$

where $g: \mathbb{R}^{k_{1}+1} \times \ldots \times \mathbb{R}^{k_{m}+1} \rightarrow B, B$ is a Banach space, $G_{i}: B \rightarrow \mathbb{R}$, $G_{i} \circ x$ is the composition of $G_{i}$ and $x(i=1, \ldots, m)$ and $\left(G_{i} \circ x\right)^{j}$ is the $j$ th iteration of $G_{i} \circ x, J^{p} y(t):=\left(y(t), y^{2}(t), \ldots, y^{p}(t)\right), y^{i}(t)$ is the $i$ th iteration of $y(t)$.

Theorem 4. Let $B$ be a Banach space, $X=\mathbb{R}^{k_{1}+1} \times \ldots \times \mathbb{R}^{k_{m}+1}$, and let $g: X \rightarrow B$ be a mapping satisfying the Lipschitz condition

$$
\|g(x)-g(y)\|_{B} \leq L\|x-y\| \quad \text { for all } x, y \in X,
$$


where $L>0,\|\cdot\|$ is the norm on $X,\|\cdot\|_{B}$ is the norm on $B$ and there is a constant $M>0$ such that $\|g(x)\|_{B} \leq M$ for all $x \in X$. Let $G_{i}: B \rightarrow R, i=$ $1, \ldots, m$, be continuously differentiable functions with $\left|D G_{i}(u) v\right| \leq M_{i}\|v\|$ for all $u, v \in B, i=1, \ldots, m$, where $D G_{i}(u) \in L(B, \mathbb{R})$ is the Fréchet derivative of $G_{i}$ at $u$ and $M_{i}>0$. If the equation (15) has a nonconstant, $T$-periodic solution $x(t)$ then

$$
T \geq 6(L S)^{-1}
$$

where

$$
\begin{array}{ll}
S=L \sum_{i=1}^{m}\left[M_{i}\left(M_{i} M-1\right)\right]\left[\left(M_{i} M\right)^{k_{i}}-1\right]^{-1} & \text { if } M_{i} M \neq 1, \\
S=\sum_{i=1}^{m} M_{i} k_{i} & \text { if } M_{i} M=1 .
\end{array}
$$

Proof. The Lemma, the condition (16), the boundedness of $g$ and $D G_{i}$ and the mean value theorem for mappings of Banach spaces imply

$$
\begin{aligned}
\int_{0}^{T} \int_{0}^{T} \| x(t)- & x(s)\left\|_{B} d t d s \leq L(T / 6) \int_{0}^{T} \int_{0}^{T}\right\| x(t)-x(s) \|_{B} d t d s \\
\leq & L(T / 6) \int_{0}^{T} \int_{0}^{T}\left[\left|\left(G_{1} \circ x\right)(t)-\left(G_{1} \circ x\right)(s)\right|+\ldots\right. \\
& +\left|\left(G_{1} \circ x\right)^{k_{1}}(t)-\left(G_{1} \circ x\right)^{k_{1}}(s)\right|+\ldots \\
& +\left|\left(G_{m} \circ x\right)(t)-\left(G_{m} \circ x\right)(s)\right|+\ldots \\
& \left.+\left|\left(G_{m} \circ x\right)^{k_{m}}(t)-\left(G_{m} \circ x\right)^{k_{m}}(s)\right|\right] d t d s \\
\leq & L(T / 6) S \int_{0}^{T} \int_{0}^{T}\|x(t)-x(s)\|_{B} d t d s .
\end{aligned}
$$

From this inequality we obtain (17).

2. Bounds for periods of difference inclusions. Consider the difference inclusion

$$
z_{i+1}-z_{i} \in F\left(z_{i}\right),
$$

where $F: U \rightarrow H^{c}, U \subset H, H$ is a Hilbert space and $H^{c}$ is the set of all compact subsets of $H$. We shall use the Hausdorff metric on $H^{c}$ defined as follows:

$$
h(A, B)=\max \{r(A, B), r(B, A)\}, \quad A, B \in H^{c},
$$

where $r(A, B)=\max _{x \in A} d(x, B), d(x, B)=\inf \{\|x-y\|: y \in B\},\|u\|=$ $(u, u)^{1 / 2}, u \in H$. 
Theorem 5. Let $H$ be a Hilbert space, $U \subset H$, and let $F: U \rightarrow H^{c}$ be a multivalued mapping satisfying the following hypotheses:

(H1) If $x, y \in U, x \neq y$ and $F(x) \cap F(y) \neq \emptyset$ then $\operatorname{diam}(F(x) \cap F(y)) \leq$ $h(F(x), F(y))$, where diam $X$ is the diameter of the set $X$.

(H2) $\quad h(F(x), F(y)) \leq L\|x-y\|$ for all $x, y \in U$, where $L>0$ is a constant.

Let $\gamma=\left\{x_{0}, x_{1}, \ldots, x_{N-1}\right\}$ be any $N$-periodic orbit of the inclusion (18) satisfying

$$
\max _{x \in \gamma} \operatorname{diam} F(x) \leq 3 L \operatorname{diam} \gamma
$$

Then

$$
N \geq 2 / 3 L
$$

Proof. Let $d=\operatorname{diam} \gamma$. Then there exist $i, j \in\{0,1, \ldots, N-1\}$ such that $d=\left\|x_{j}-x_{i}\right\|$. Assume $j>i$. Then

(20) $d^{2}=\left(x_{j}-x_{i},\left(x_{j}-x_{j-1}\right)+\left(x_{j-1}-x_{j-2}\right)+\ldots+\left(x_{i+1}-x_{i}\right)\right)$.

The mapping $F$ is compact valued and therefore there exist $u_{1} \in \gamma$ and $y_{1} \in F\left(u_{1}\right)$ such that

$$
\left(x_{j}-x_{i}, y_{1}\right)=\max _{z \in F\left(u_{1}\right)}\left(x_{j}-x_{i}, z\right)=\max _{x \in \gamma} \max _{y \in F(x)}\left(x_{j}-x_{i}, y\right) .
$$

Since $x_{k+1}-x_{k} \in F\left(x_{k}\right)$ for $k=i, i+1, \ldots, j-1$ we obtain from (20), (21)

$$
\begin{aligned}
d^{2} & \leq \max _{y \in F\left(x_{j-1}\right)}\left(x_{j}-x_{i}, y\right)+\ldots+\max _{y \in F\left(x_{i}\right)}\left(x_{j}-x_{i}, y\right) \\
& \leq N\left(x_{j}-x_{i}, y_{1}\right) .
\end{aligned}
$$

Obviously

$$
x_{i}-x_{j}=\sum_{m=0}^{i-1}\left(x_{m+1}-x_{m}\right)+\sum_{n=j}^{N-2}\left(x_{n+1}-x_{n}\right)+x_{0}-x_{N-1} .
$$

There exist $u_{2} \in \gamma$ and $y_{2} \in F\left(u_{2}\right)$ such that

$$
\left(x_{i}-x_{j}, y_{2}\right)=\max _{z \in F\left(u_{2}\right)}\left(x_{i}-x_{j}, z\right)=\max _{x \in \gamma} \max _{y \in F(x)}\left(x_{i}-x_{j}, y\right) .
$$

From (23), (24) we obtain

$$
\begin{aligned}
d^{2} \leq & \sum_{m=0}^{i-1} \max _{y \in F\left(x_{m}\right)}\left(x_{i}-x_{j}, y\right)+\sum_{n=j}^{N-2} \max _{y \in F\left(x_{n}\right)}\left(x_{i}-x_{j}, y\right) \\
& +\max _{y \in F\left(x_{N-1}\right)}\left(x_{i}-x_{j}, y\right) \\
\leq & N\left(x_{i}-x_{j}, y_{2}\right)=N\left(x_{j}-x_{i},-y_{2}\right) .
\end{aligned}
$$

The inequalities (22), (25) imply

$$
2 d^{2} \leq N\left\|x_{j}-x_{i}\right\|\left\|y_{1}-y_{2}\right\| .
$$


We shall prove that

$$
\left\|y_{1}-y_{2}\right\| \leq 3 L\left\|x_{j}-x_{i}\right\|
$$

If $F\left(u_{1}\right)=F\left(u_{2}\right)$ then $\left\|y_{1}-y_{2}\right\| \leq \operatorname{diam} F\left(u_{1}\right)$. By the hypothesis (H3) we have $\operatorname{diam} F\left(u_{1}\right) \leq 3 L \operatorname{diam} \gamma=3 L\left\|x_{j}-x_{i}\right\|$, i.e. (27) holds. Let $F\left(u_{1}\right) \neq F\left(u_{2}\right)$. There exist $z_{1}, z_{2} \in F\left(u_{1}\right), v_{1}, v_{2} \in F\left(u_{2}\right)$ such that $h\left(F\left(u_{1}\right), F\left(u_{2}\right)\right)=\max \left\{d_{1}, d_{2}\right\}$, where $d_{1}=\left\|z_{1}-v_{1}\right\|=r\left(F\left(u_{2}\right), F\left(u_{1}\right)\right)$, $d_{2}=\left\|z_{2}-v_{2}\right\|=r\left(F\left(u_{1}\right), F\left(u_{2}\right)\right)$.

First we assume that $F\left(u_{1}\right) \cap F\left(u_{2}\right)=\emptyset$. Then obviously

$$
\begin{aligned}
\left\|y_{1}-v_{1}\right\| & \leq\left\|z_{1}-v_{1}\right\|=d_{1} \leq h\left(F\left(u_{1}\right), F\left(u_{2}\right)\right), \\
\left\|y_{2}-z_{2}\right\| & \leq\left\|z_{2}-v_{2}\right\|=d_{2} \leq h\left(F\left(u_{1}\right), F\left(u_{2}\right)\right), \\
\left\|v_{1}-z_{2}\right\| & \leq d_{2} \leq h\left(F\left(u_{1}\right), F\left(u_{2}\right)\right) .
\end{aligned}
$$

From these inequalities and the hypothesis (H2) we obtain

$$
\left\|y_{1}-y_{2}\right\| \leq 3 h\left(F\left(u_{1}\right), F\left(u_{2}\right)\right) \leq 3 L\left\|u_{1}-u_{2}\right\| \leq 3 L\left\|x_{j}-x_{i}\right\| .
$$

Let now $F\left(u_{1}\right) \cap F\left(u_{2}\right) \neq \emptyset$. If $y_{1}, y_{2} \in F\left(u_{1}\right) \cap F\left(u_{2}\right)$ then from (H1), (H2) it follows that

$$
\begin{aligned}
\left\|y_{1}-y_{2}\right\| & \leq \operatorname{diam}\left(F\left(u_{1}\right) \cap F\left(u_{2}\right)\right) \leq h\left(F\left(u_{1}\right), F\left(u_{2}\right)\right) \\
& \leq 3 L\left\|u_{1}-u_{2}\right\| \leq 3 L\left\|x_{j}-x_{i}\right\| .
\end{aligned}
$$

If $y_{1} \in F\left(u_{1}\right) \backslash F\left(u_{2}\right)$ and $y_{2} \in F\left(u_{2}\right) \backslash F\left(u_{1}\right)$ then (28) and (29) obviously hold and (H1) implies that

$$
\left\|v_{1}-z_{2}\right\| \leq \operatorname{diam}\left(F\left(u_{1}\right) \cap F\left(u_{2}\right)\right) \leq h\left(F\left(u_{1}\right), F\left(u_{2}\right)\right) .
$$

Therefore (31), and hence (27) holds. If $y_{1} \in F\left(u_{1}\right) \backslash F\left(u_{2}\right)$ and $y_{2} \in$ $F\left(u_{1}\right) \cap F\left(u_{2}\right)$ then (28) obviously holds and (H1) yields

$$
\begin{aligned}
\left\|y_{2}-z_{2}\right\| & \leq \operatorname{diam}\left(F\left(u_{1}\right) \cap F\left(u_{2}\right)\right) \leq h\left(F\left(u_{1}\right), F\left(u_{2}\right)\right), \\
\left\|v_{1}-z_{2}\right\| & \leq \operatorname{diam}\left(F\left(u_{1}\right) \cap F\left(u_{2}\right)\right) \leq h\left(F\left(u_{1}\right), F\left(u_{2}\right)\right) .
\end{aligned}
$$

Therefore (27) again holds. The inequalities (26), (27) yield

$$
2 d^{2} \leq 3 L N\left\|x_{j}-x_{i}\right\|^{2}=3 L N d^{2}
$$

and this implies (19).

EXAMPLE 1 . Let $f:[a, b] \rightarrow \mathbb{R}$ be a countinuously differentiable function, $a<b, c \in(a, b), A=[0, c-\delta] \cup[c+\delta, b], U=A \cup\{c\}$. Define a multivalued mapping $F: U \rightarrow \mathbb{R}^{c}\left(\mathbb{R}^{c}\right.$ is the set of all compact subsets of $\left.\mathbb{R}\right)$ as follows: $F(x)=\{f(x)-x\}$ if $x \in A$ and $F(c)=I_{\varepsilon}:=[\alpha-\varepsilon, \alpha]$, where $\alpha=$ $f(c)-c$ and $0<\varepsilon$. If $x, y \in A$ then the mean value theorem implies that $h(F(x), F(y))=|f(x)-x-(f(y)-y)| \leq p|x-y|$, where $p=\max _{x \in A}\left|f^{\prime}(x)\right|+$ 1. If $x \in A$ then there exists $u \in I_{\varepsilon}$ such that $h(F(x), F(c))=|f(x)-x-u|$. Define $k(x, y)=\left|(f(x)-x-u)(x-c)^{-1}\right|$ for $(x, y) \in A \times I_{\varepsilon}$. Then $k$ has a maximum $q \geq 0$ and therefore $|f(x)-x-u| \leq q|x-c|$ for all $x \in A$. Thus 
we have proved that $h(F(x), F(c)) \leq q|x-c|$ for all $x \in A$. If $L=\max (p, q)$ then $h(F(x), F(y)) \leq L|x-y|$ for all $x, y \in U$, i.e. $F$ satisfies (H2). Since $F$ is single-valued on $A$ the hypothesis (H1) is trivially satisfied. By Theorem 5 if $\gamma$ is an $N$-periodic trajectory of (18) and $\operatorname{diam} \gamma \geq \varepsilon / 3 L$ then $N \geq 2 / 3 L$. We remark that if $x_{i} \neq c$ and $x_{i} \notin(c-\delta, c+\delta)$ then $x_{i+1}-x_{i} \in F\left(x_{i}\right)$ if and only if $x_{i+1}=f\left(x_{i}\right)$.

EXAMPLE 2. Let $f:[a, b] \rightarrow \mathbb{R}$ be a continuously differentiable function, $a<b, c_{1}, c_{2} \in(a, b), c_{1}<c_{2}, f\left(c_{1}\right)-c_{1}=f\left(c_{2}\right)-c_{2}, A=\left[a, c_{1}-\delta\right]$ $\cup\left[c_{1}+\delta, c_{2}-\delta\right] \cup\left[c_{2}+\delta, b\right], 0<\delta<c_{1}, \delta<c_{2}-c_{1}, \delta<b-c_{2}, U=$ $A \cup\left\{c_{1}\right\} \cup\left\{c_{2}\right\}$. Define $F: U \rightarrow \mathbb{R}^{c}$ by $F(x)=\{f(x)-x\}$ if $x \in A$, $F\left(c_{2}\right)=I_{\varepsilon}=[\beta-\varepsilon, \beta], F\left(c_{1}\right)=I_{3 \varepsilon}=[\beta-3 \varepsilon, \beta], \beta=f\left(c_{1}\right)-c_{1}$. As above one can show that (H2) is satisfied, where $L=\max _{x \in A}\left|f^{\prime}(x)\right|+1$. Obviously, if $x \in U, x \neq c_{1}, c_{2}, F(x) \cap F\left(c_{i}\right) \neq \emptyset(i=1,2)$ then $\operatorname{diam}\left(F(x) \cap F\left(c_{i}\right)\right)=0$ and $\operatorname{diam}\left(F\left(c_{1}\right) \cap F\left(c_{2}\right)\right)=\varepsilon<h\left(F\left(c_{1}\right), F\left(c_{2}\right)\right)=2 \varepsilon$, i. e. (H1) is satisfied. Theorem 5 implies that if $\gamma$ is an $N$-periodic trajectory and $\varepsilon \leq L$ diam $\gamma$ then $N \geq 2 / 3 L$.

\section{References}

[1] S. Busenberg and M. Martelli, Bounds for the periodic orbits of dynamical systems, J. Differential Equations 67 (1987), 359-371.

[2] -, 一, Better bounds for periodic orbits of differential equations in Banach spaces, Proc. Amer. Math. Soc. 86 (1986), 376-378.

[3] S. Busenberg, D. Fisher and M. Martelli, Minimal periods of discrete and smooth orbits, Amer. Math. Monthly 96 (1989), 5-17.

[4] A. Lasota and J. A. Yorke, Bounds for periodic solutions of differential equations in Banach spaces, J. Differential Equations 10 (1971), 83-91.

[5] T. Y. Li, Bounds for the periods of periodic solutions of differential delay equations, J. Math. Anal. Appl. 49 (1975), 124-129.

[6] Z. Nitecki, A periodic attractor determined by one function, J. Differential Equations 29 (1978), 214-234.

[7] W. Słomczyński, Bounds for periodic solutions of difference and differential equations, Comment. Math. 26 (1986), 325-330.

[8] J. A. Yorke, Periods of periodic solutions and Lipschitz constant, Proc. Amer. Math. Soc. 22 (1969), 509-512.

MATHEMATICAL INSTITUTE

SLOVAK ACADEMY OF SCIENCES

ŠTEFÁNIKOVA 49, 81473 BRATISLAVA

CZECHOSLOVAKIA 